\title{
The use of allomorphism for the access control service in OSI management environment
}

Ramos, A. M.

Universidade do Vale do Itajaí - Univali

University of Vale do Itajai - Univali

Faculdade de Ciências Exatas - Curso de Computação

Rua Patricio Antonio Teixeira, s/n - Cep 88160-000 Biguaçu/SC/Brazil - Phone/Fax: 55048 2314091E-mail:moraes@inf.ufsc.br

Specialski, E. S.

Universidade Federal de Santa Catarina

Federal University of Santa Catarina State - UFSC

Curso de Pós-Graduação em Ciência da Computação

Cx Postal 476 - Campus Universitário - Cep 88040-900 Florianópolis/SC/Brazil Phone 550482319738 -

Fax 550482319770 -E-mail: beth@inf.ufsc.br

\begin{abstract}
This paper proposes the use of Allomorphism for the access control service in OSI management environment, with no Access Control Function implementation (ISO10164-9, 1990), which is responsible for this service. The Allomorphism is a powerful SMI resource that makes the OSI Model very strong and flexible. This strategy reduces the overhead introduced by the access control function on OSI management systems without expose the management to arbitrary use of its resources. The Allomorphism allows to define different access views to the management resources according to security requirements. In this context, this work presents the security requirements in networks management systems, the OSI Access Control Function Model and its disadvantages, the idea of Allomorphism and, finally, how to implement it to access control.
\end{abstract}

Keywords

Allomorphism, access control service, OSI management environment 


\section{INTRODUCTION}

The scope of this paper is the OSI management environment, the access control service and its implementations alternatives using the access control function (ISO10164-9, 1990) and the allomorphism. The paper is designed to encompass the following objectives:

- To emphasize the vulnerabilities created for the network by the management systems and, consequently, the importance of access control service;

- To analyze the access control function (ISO10164-9, 1990) and their particularities;

- To analyze the allomorphism concept and its flexibility;

- To propose an alternative implementation for the access control service in OSI management environment which is based on the use of allomorphism;

- And, finally, to analyze the impact of the access control service implementation using the allomorphism.

Computer Network Management can provide benefits to the network system such as to control faults, to evaluate performance, to identify systems violation, to exercise control over configuration, to determine and allocate costs and charges for the use of the network resources, and others. However, some security conditions need to be implemented to control unauthorized people to access the network resources through the management system application itself.

Although management systems make the administration process of the network easier for the administrator, they also create a vulnerable point which can be used to manipulate extremely important information for the good functionality of the network. When management exists more points to attack the system and more vulnerability are created, more possibility to compromise the net and its systems. These and many other weaknesses would not exist if a management system was not implemented (De Lucca, 1993).

So, the OSI management Model (ISO7498-4, 1989) defined the security functional area which aims to control security mechanisms, facilities and services in such a way that it will protect the network resources as well as (its own) the management application itself against system violations. In order to achieve all the requirements, three security management functions were defined to support the functional area; which are Security Alarm Reporting Function (ISO10164-7, 1991); Security Audit Trail Function (ISO10164-8, 1990); Objects and Attributes for Access Control (ISO10164-9, 1990).

The Security Alarm Reporting Function notifies wrong operations in security mechanisms and services; and attempts to violate the network or management security. The Security Audit Trail Function records all potential events related to security in a $\log$ file for security audit. The Objects and Attributes for Access Control Function protects the management resources against nonauthorized access. 
One or more access control mechanisms are implemented to ensure only access to specific resources by authorized users.

The alternative proposed in this article is resulted from the access control function (ISO10164-9, 1990) implementation in the UFSC's network management framework (Ramos, 1994).

\section{OBJECTS AND ATTRIBUTES FOR ACCESS CONTROL FUNCTION}

The Objects and Attributes for Access Control Function (ISO10164-9, 1990) has specific access control within the management system. Once an application management access is obtained, all associations among agent and management processes and all management operations on managed objects store in MIB are controlled by the Access Control Function and are managed by the Security Alarm Reporting Function and Security Audit Trail Function.

Many access levels may be needed to be implemented:

- Read/write access, read only or no access at all;

- Access only for some objects; and

- No permission to have a management association established.

Nonauthorized entities should not have access to management operations; and, all management warnings including, security alarm, should only be sent to authorized users.

In order to accomplish this, the security authority must define an access control policy for the management resources. These rules define: what kind of management information need controlled access; those who may manipulate them and the conditions to do so; and, what rules will control the access to management information.

Objects to be managed and their attributes model the management resources. Therefore when management objects and their attributes are access controlled so are the network resources automatically access controlled.

The management information which need access control is associated to another object, exclusive to access control, called target. The target has attributes to represent information and rules of access control to be applied to its associated object, which means the information that the object represents. For example, a target may represent a managed object, an attribute of managed object, an attribute value or even a management action.

The access control policy for resources management consists of specification of target objects that represent information and rules. There are two types of access control for OSI management:

- For association of management applications, to guarantee that non-authorized entities initiator will establish management associations;

- For management operations, to guarantee that operations will be made by authorized entities but with restrictions related to time, operation type, resources and information involved. 
The Access Control Function has the basic model presented in Figure 1, with the following functions: Access Control Enforcement Function (AEF) and Access Control Decision Function (ADF).

The AEF function receives the access request made by an user, also called initiator, selects the access control parameters for that request and transfer these parameters to the ADF function.

The ADF function receives the access control parameters from the AEF function and decides if the access request is valid or not. For this validation, the ADF function compares the request parameters received from the AEF function with the rules of the access control policy defined for the network. The request is validated if it follows the rules. Otherwise, the request is rejected. The decision is passed back to the $\mathrm{AEF}$ function. If the request is rejected the $\mathrm{AEF}$ function will also receive the rejection instructions which can be just an access denied or even an instruction to abort the connection.

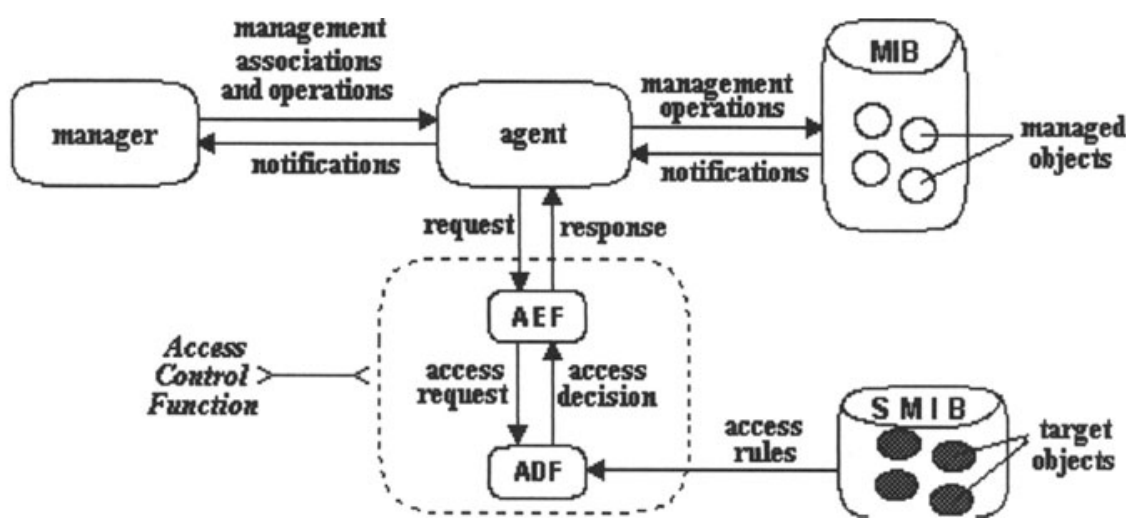

Figure 1 - Access Control Function Model

The OSI Management Information Model is based on Object Oriented Paradigm where objects are logic abstraction of physical resources. Objects can be modeled using the paradigms: class, attribute, encapsulation, inheritance, multiple inheritance, and allomorphism as defined by SMI (Structure Management Information) (ISO10165-1,2,3,4). Objects are organized following a hierarchy in a Management Information Base (MIB).

The information of access control is also stored with object oriented paradigm using objects of access control called target objects. Each management resource, or management object, which needs security is associated to a target object. All target objects are in a Security Management Information Base (SMIB) defined at conceptual level. The SMIB can be integrated partially or totally to MIB. 


\section{ALLOMORPHISM}

Allomorphism allows an instance of a class to behave like an instance of one or more other classes. This feature provides more power and flexibility to the OSI Management Model (Pavlou, 1993).

In general, the OSI Information Model allows members of arbitrary classes, derived or not, to be related allomorphically if they have a compatible behave. The object that emulates completely behave and characteristics of a member of another management object class is said to be allomorphic. Allomorphism is used by management systems to allow a better interoperability of manager and managed objects.

For example, when the management system is updated to a new version, the objects needed to be extended to include the new features. By definition, allomorphic classes must provide management of new systems and accommodate their new additional features in the current system.

Allomorphic classes, when derived, are called allomorphic subclasses and present similar behave to their superclasses. Different versions of a resource is an example o allomorphic class. Allomorphic subclasses are a type of specialization of superclasses where the inheritance characteristics from the superclass must be restriction in the allomorphic subclass (ISO10165-1, 1991).

Allomorphic inheritance shows restrictions to attributes, actions, and notifications. Regarding attributes, any acceptable value in the subclass must also to be acceptable in the superclass. The following restrictions apply for the inherited actions of the subclass:

- New optional and response parameters may be added to the action argument if the superclass is able to be extended;

- New mandatory parameters may be defined for the action argument if default values are defined to be assumed when nothing is specified;

- New necessary parameters may not be added - because they must be present at the execution time of the action;

- New actions can be defined; however, they can never be invoked by a system that doesn't accept the new class.

Restrictions of notifications for inherited events refer to inclusion of new response parameters and extension of value limits for existent arguments that are neither one allowed.

Figure 2 shows the inheritance tree that starts with the root class which is the superclass of all other classes. Net and system are subclasses of the root class. Net has only one subclass which is sub-net. On the other hand, system has a subclass equipment with 3 other subclasses: switch, router and hub.

Figure 3 shows the structure when the router subclass is defined as allomorphic to its superclass equipment. One class can be allomorphic to one or more classes. In the allomorphic class is important to have an attribute (allomorphsClassesDefault) that identify the default classes which has an allomorphic behavior. At the moment of the managed object creation, the allomorphs attribute of TOP Class receive the value of this attribute (allomorphsClassesDefault). 


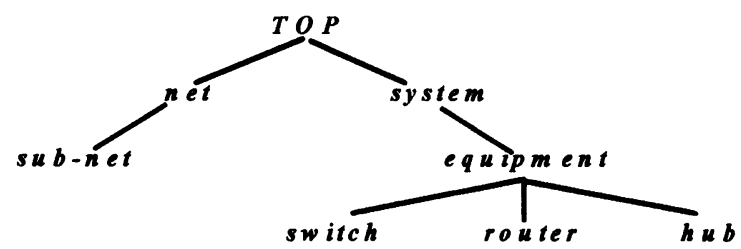

Figure 2 - Example of Hierarchy

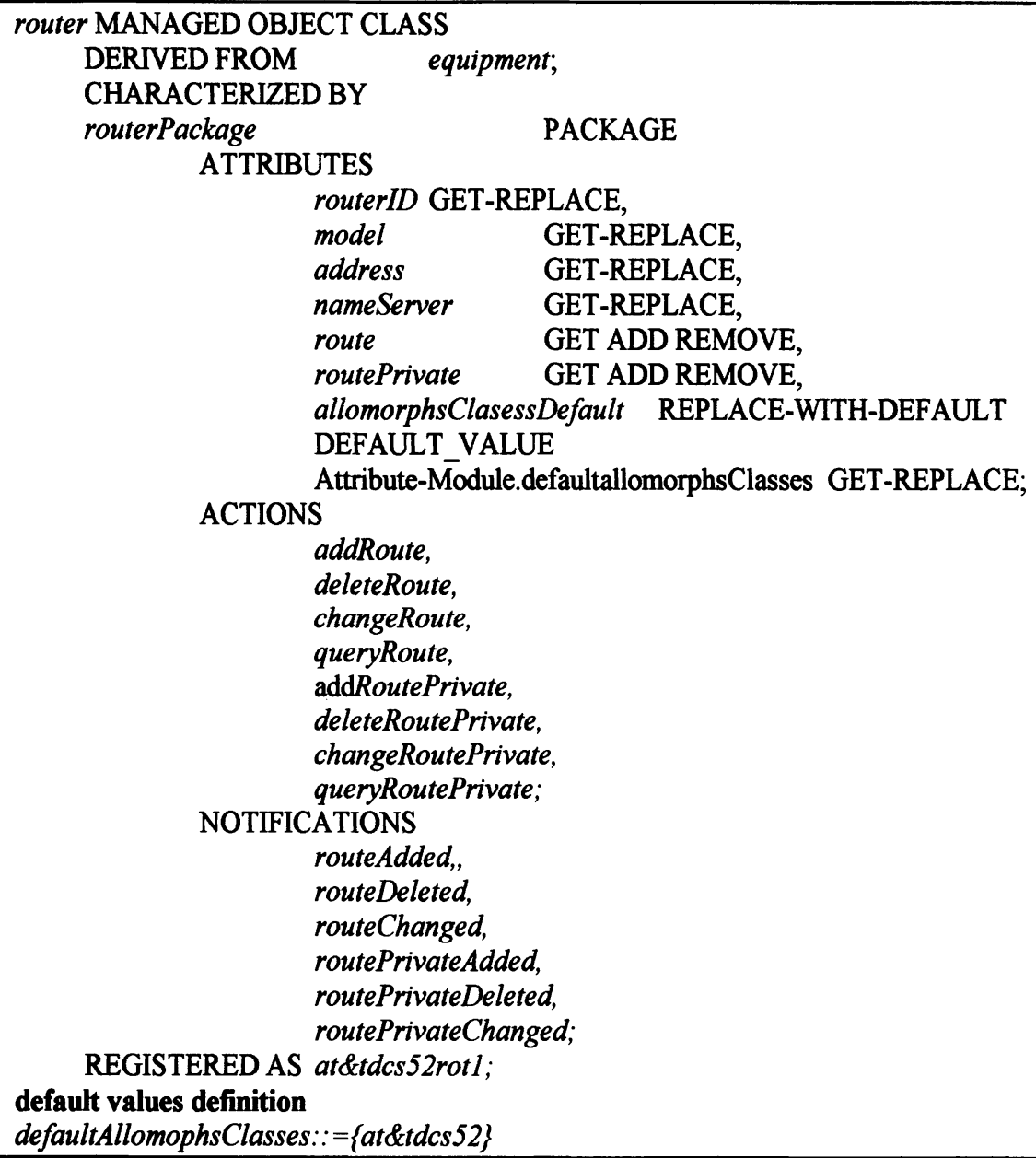

Figure 3 - Definition of the Router Class

The router subclass is at the same time derived and allomorphic to its superclass equipment. One instance of the class router can behave like an instance of the equipment class as well as an instance of the router class itself.

If the router subclass identifies equipment and system superclasses as members of its allomorphsClassesDefault, it is possible to operate an instance of the router class as an instance of the system and equipment classes. However, the allomorphic resource is not transient. If the router class is allomorphic to the equipment class, 
and the if equipment class identifies the system class in its allomorphic set attribute, it doesn't mean that one instance of the router class can behave like an instance of the system class.

Allomorphism also allows instances of arbitrary classes to present same the behave. One example is shown in Figure 4 where the switch class is defined as allomorphic to the router class. This hypothetical case would be used to have a switch imitating a router in tests of a network system.

It is important to remind, however, that Allomorphism and Polymorphism have different concepts. Polymorphism is an object oriented paradigm and allows a function to behave in different ways. Allomorphism, on the other hand, is a resource of OSI Management Information Model which allows an instance of a class to imitate the behave of an instance of one or more other classes.

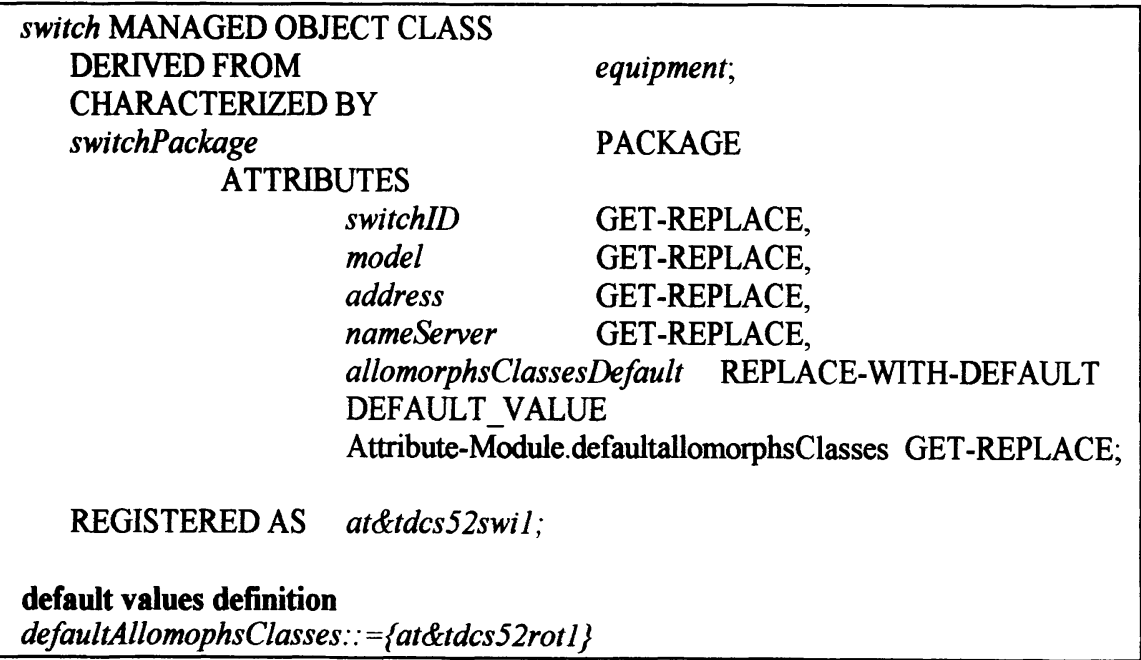

Figure 4 - Definition of the Switch Class

\section{ALLORMORPHISM AND THE ACCESS CONTROL FUNCTION}

The Access Control Function (ISO10164-9, 1990) guarantees associations and management operations at run time. When an OSI management system is activated, the access control function will be called by the application process in order to have an access decision every time a) an association among application processes (agents or mangers) is established; or, b) a management operation (create, delete, action, set and get) is made on an object stored in the MIB.

The access decision process calls the AEF function which calls the ADF function. The later makes inference on the SMIB security base. It accesses the target object associated, compares the access request with the protection rules defined to the target object, and returns the decision access to the AEF function which will repass the decision to the agent application processed as shown previously in Figure 1. 
It is noticeable that the Access Control Function Model requires a well organized structure, however, flexible and of easy maintenance of the SMIB base in order to make a fast decision access.

The management system already makes an overhead operation to the network when it adds the security service. When more traffic is created, regular and management applications processes will become slower. The access decision for a local process doesn't generates more traffic in the network, but the OSI Model is based in CMIP (Common Management Information Protocol) protocol, which means that it will be waiting for an answer. The OSI Model is connection oriented; so, there will always be a remote application process, the manager, waiting for a management operation or a management association.

This paper proposes the use of allomorphism for the access control service instead of OSI Model Access Control Function (ISO10164-9, 1990). Allomorphism is a property of the OSI Management Model which allows the network manager to define relationships among classes and release them to users according to the management system needs.

According to (Klerer, 1993), allomorphism allows view definitions (or masks) of managed object classes. The network manager may hide some attributes and events to a group of users. An allomorphic class can be defined from a managed object class with some characteristics left apart. The user will access only the redefined characteristics in the allomorphic class.

Figures 5, 6 and 7 show a new representation of the equipment class presented in Figure 2. The equipment super-class will have two new classes: OperatorRouter and UserRouter, to implement the router equipment in the access control service.

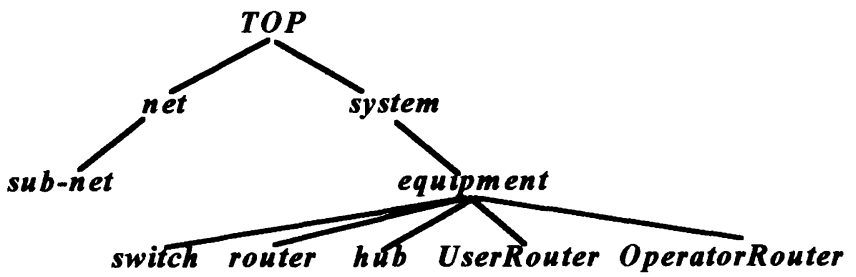

Figure 5 - Hierarchy for the Access Control Service

\begin{tabular}{|c|c|c|}
\hline \multicolumn{2}{|c|}{ UserRouter MANAGED OBJECT CLASS } & \\
\hline DERIVED FROM & & equipment; \\
\hline CHARACTERIZED & BY & \\
\hline UserRouterPackage & & PACKAGE \\
\hline ATTRIE & UTES & \\
\hline & routerID & GET, \\
\hline & model & GET, \\
\hline & address & GET, \\
\hline & nameServer & GET, \\
\hline & route & GET; \\
\hline ACTIOI & & \\
\hline REGISTERED AS & $\begin{array}{l}\text { queryRoute; } \\
\text { at\&tdcs52rot12; }\end{array}$ & \\
\hline
\end{tabular}

Figure 6 - Definition of the UserRouter Class 


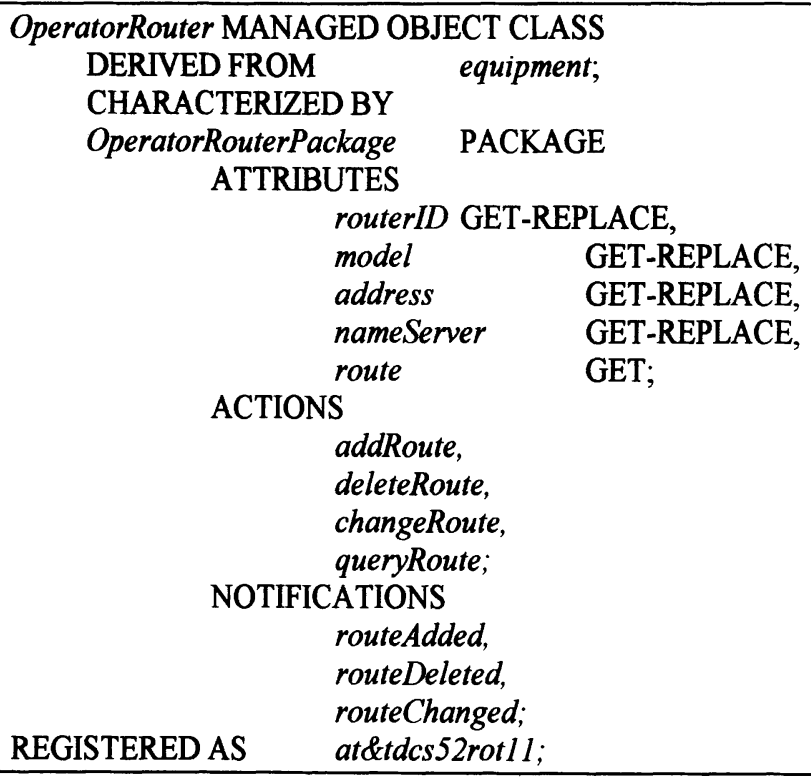

Figure 7 - Definition of the OperatorRouter Class

One instance of the router class can behave like an instance of one of the two classes: OperatorRouter or UserRouter. But the router class definition needs to contain the OperatorRouter and the UserRouter values in its allomorphsClassesDefault attribute to hold the allomorphic characteristic as seen in Figure 8.

Router, OperatorRouter and UserRouter classes model the same physical resource (Figure 9). Each class represents one different type of modeling for different users.

One given user of the router resource will access only the resources of the modeled object referred by the class defined for him/her. The network operators will only manipulate the instances of the modeled classes for the Operator group. Regular users of the management system will manipulate instances with behave like the ones in the User group class. The network manager will manipulate, on the other hand, all instances and they may have allomorphic behave of all kinds of groups.

The router class, as seen previously in Figure 8 , belongs to the Manager group in Figure 9. The instances of this class with all their attributes can be totally seen by the manager group, and the instances can also behave like their allomorphic classes.

The Operator group has the OperatorRouter class associated to its group and can see the router through the modeled class. Close to all resources associated to the router can be available to this group, except the information that manipulates the private routes which are exclusive to the network manager group.

The UserRouter class is the simplest. This class only allows the User group to execute the action QueryRoute. No other action will be available for this group. In addition, this group has neither authorization to receive notification or to alter attribute values for this router. 
router MANAGED OBJECT CLASS

DERIVED FROM CHARACTERIZED BY routerPackage

equipment;

ATTRI

\section{PACKAGE}

ATTRIBUTES

routerID GET-REPLACE,

model

GET-REPLACE,

address

GET-REPLACE,

nameServer

GET-REPLACE,

route

GET ADD REMOVE,

routePrivate GET ADD REMOVE,

allomorphsClassesDefault REPLACE-WITH-DEFAULT

DEFAULT_VALUE,

ACTIONS

Attribute-Module.defaultallomorphsClasses GET-REPLACE;

addRoute,

deleteRoute,

changeRoute,

queryRoute,

addRoutePrivate,

deleteRoutePrivate,

changeRoutePrivate,

NOTIFICATIONS

queryRoutePrivate;

\section{REGISTERED AS}

routeAdded,

routeDeleted,

routeChanged,

routePrivateAdded,

routePrivateDeleted,

routePrivateChanged;

default values definition

defaultAllomophsClasses: $:=\{a t \& t d c s 52$, at\&dtcs52rot 11, at\&dtcs52rot 12\}

Figure 8 - Definition of the Router Allomorphic Class

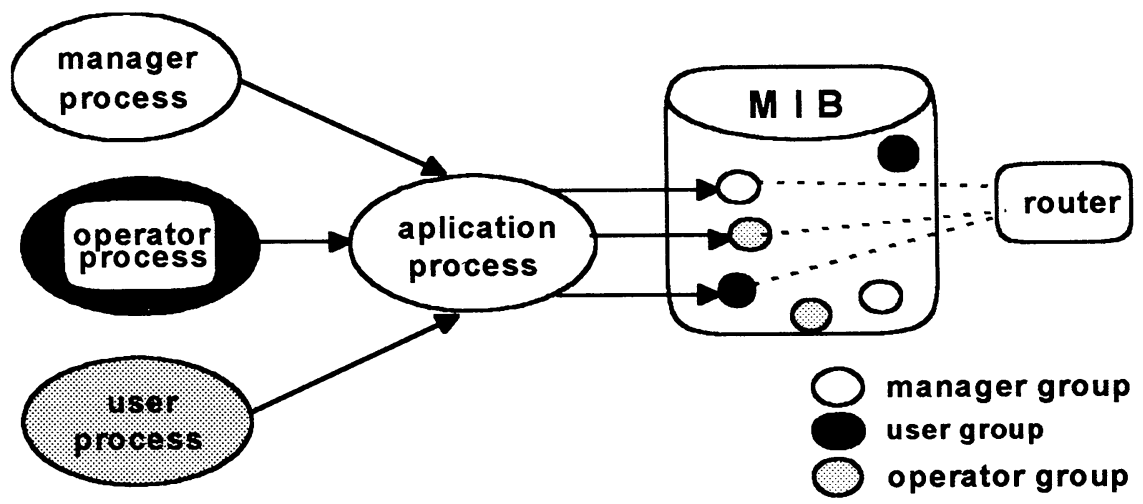

Figure 9 - Masks for Access Control 
A management application process must provide its group and its access level to the agent application process before executing an operation on a router class instance. The management application process uses a filter to choose the appropriate access mask that will be applied. One can conclude then that an instance of the router class will exhibit allomorphic behavior of an instance of the class compatible with the management application process and all its access rights.

The access control service can be implemented by defining several modeled classes of the same resource ( access masks: router, OperatorRouter, UserRouter, etc.), one for each group of users.

Definition of user groups and classes of the managed objects for these groups are rules in the access control policy which is defined by the network manager and is implemented in the management system. Information about the different access control policy models for management systems can be found in (Ramos, 1994).

Access control policies can be applied to one user (Individual-based), user groups (Group-based), or through security levels associated to management information which need protection (MultilevelSecurity).

When implementing the access control service by allomorphism, the one responsible for the system security must consider the following regarding the access control policy:

- Identify elements in the management system that need access protection; such as: application processes at association level and managed objects at management operation level;

- Identify the best access control policy model to help the system needs; that means: decide if the policy must be applied to each user individually, to a group of users, or to create security levels for accesses;

- Create resource views (using masks) for different group of users protecting important information.

The access control service implemented with allomorphism concepts in an OSI management system eliminates the Access Control Function (ISO10164-9, 1990) need because there is no access control at operational level in the management system. This process is transparent to the user since it is implemented in the system. A user or a group of users will see only a set of management information that is defined by the access mask defined for him.

When a user attempts to make an access not defined in his/her domain, the object will reject the access through its behavior because it does not accept such resource and its characteristics are not extended. This is guaranteed with the filter mechanism.

Although the access control service is implemented at the system management implementation, it is not static for the whole system life due to its allomorphism that provides flexibility in the system. It is possible to define a new access control policy while creating new user groups and new masks for resources. To do so, new allomorphic classes are defined according to system protection needs. 


\section{CONCLUSION}

Since computer network became an important and strategic process in private and public institutions, it is of extremely importance to present a system with security and efficiency. To maintain a functional network system at acceptable level, it is necessary to implement a network management system applications.

Management system provides many benefits to a network administration, however, like any other computing system, it is subject to security violation and requires a security service.

Application management and security services are extra processes in the network and cause some overhead in its performance. They need to be implemented together to guarantee the network security.

Not only authentication mechanisms, cryptography and passwords control are important in a security service, but specially an access control system. The access control function is fundamental as a control in the management system with protection of management resources.

The Access Control Function Model (ISO10164-9, 1990) guarantees associations and management operations at run time. As presented in this paper, this function presents a complex with many messages exchanges between the AEF and ADF functions, which cause an overhead in the management application, and makes many times its use not acceptable. Due to this overhead, many administrators deactivate the access control function to release the system for better performance, however, they expose the system to violations.

This paper presents another option which is very practical and uses the allomorphism to implement the access control service in a flexible and simpler way and with less impact in the OSI management.

Another advantage of this implementation is a reduction in the overhead with the access decision process. When we define the access control policy and the different access masks, we transfer the access decision to the management system, while in the OSI function they are made at execution time.

The responsibility is then is passed to the one who implements the system who is also responsible for creating the allomorphic classes. And so, he/she will guarantee that a user and group of users will access only those resources defined in their domain.

Allomorphism also allows different access control policies in management systems (Individual-based, Group-based, Multilevel-based, etc.). Using definition of new allomorphic classes, it is possible to create new user groups, new access masks, define new access control policy according to protection of access in the OSI management systems. 


\section{REFERENCES}

CCITT X.720. (1992) CCITT - Recommendation X.720 - Information Thecnology - Open Systems Interconnection - Structure of Management Information: Management Information Model.

CCITT X.722 (1992) CCITT - Recommendation X.722 - Information Thecnology

- Open Systems Interconnection - Structure of Management Information: Guidelines for the Definition of Managed Objects.

ISO7498-4. (1989) ISO/IEC 7498-4 - Information Processing Systems - Open Systems Interconnection - Basic Reference Model - Part 4: Management Framework.

ISO10164-7. (1991) ISO/IEC DIS 10164-7 - Information Technology - Open Systems Interconnection - Systems of Management - Part 7: Security Alarm Reporting Function.

ISO10164-8. (1990) ISO/IEC DIS 10164-8 - Information Technology - Open Systems Interconnection - Systems of Management - Part 8: Security Audit Trail Function.

ISO10164-9. (1991) ISO/IEC CD 10164-9 - Information Technology - Open Systems Interconnection - Systems of Management - Part 9: Objects and Attributes for Access Control.

ISO10165-1. (1991) ISO/IEC DIS 10165-1 - Information Thecnology - Open Systems Interconnection - Structure of Management Information: Management Information Model.

ISO10165-2. (1989) ISO/IEC DIS 10165-2 - Information Thecnology - Open Systems Interconnection - Structure of Management Information: Definition of Management Information Model.

ISO10165-3. (1989) ISO/IEC DIS 10165-3 - Information Thecnology - Open Systems Interconnection - Structure of Management Information: Definitions of Management Attributes.

ISO10165-4. (1991) ISO/IEC DIS 10165-4 - Information Thecnology - Open Systems Interconnection - Structure of Management Information: Guidelines for the Definition of Managed Object.

KLERER, M. (1993) Systen Management Information Modeling, IEEE Communications Magazine.

DE LUCCA, J. E. (1994) Arquitetura para Segurança em Gerência de Redes, Trabalho Individual - CPGCC UFSC, Santa Catarina.

PAVLOU, G. et alli. (1993) A Generic Management Information Base Browser, The ISO Development Environment, Versão 8.0, University College London.

RAMOS, A. M. (1994) Interface de Controle de Acesso para o Modelo de Gerenciamento OSI, Dissertação de Mestrado - CPGCC UFSC, Santa Catarina. 


\section{BIOGRAPHY}

RAMOS, ALEXANDRE MORAES, M.Sc., is an Assistant Professor Graduate of Computer Science Department at University of Vale do Itajai in Santa Catarina, Brazil. At the same time, he works on network management at Water Resources and Meteorological Integrated Centre of Santa Catarina, Brazil. His research interests include network management, telecommunications management, and information technology.

SPECIALSKI, ELIZABETH SUELI has been working on computer network research and development since 1980, mainly in network management. She has been teaching on Computer Science Pos-Graduation Program at Federal University of Santa Catarina, Brazil since 1989. She heads a workgroup which designs and develops a network management platform and provides network consulting services. She also heads a group which works for Human Resources formation in Internet administration. 